\title{
Effects of manufacturing type on control performance of multiple tuned mass dampers under harmonic excitation
}

\author{
O. Araz ${ }^{* 1}$, V. Kahya ${ }^{2}$ \\ ${ }^{1}$ Gümüşhane University, Department of Civil Engineering, 29100, Gümüşane, Turkey \\ ${ }^{2}$ Karadeniz Technical University, Department of Civil Engineering, 61080, Trabzon, Turkey
}

\begin{abstract}
Using different optimization techniques, the optimum characteristics of multiple tuned mass dampers (MTMD) for reducing the dynamic response of primary structure due to various excitations are studied by many researchers. In this paper, the five practical MTMD models are considered which consist of different combinations of the mass, damping, stiffness coefficient and damping ratio in the MTMD. The criteria selected for the optimality is the minimization of the maximum value of the displacement dynamic magnification factor (DMF) of the primary structure with the MTMD system under external harmonic excitation. The optimum parameters of the MTMD systems are investigated using Sequential Quadratic Programming (SQP). Results obtained illustrate that the optimum MTMD-1 and MTMD-4 have nearly the same control performance and provide higher effectiveness than all the other types of MTMDs of the same mass ratio in reducing the displacement response of primary structure. Finally, a robustness analysis of optimally designed MTMD systems to investigate their performance under errors in the tuned frequencies is also performed in detail.
\end{abstract}

\section{Keywords}

Dynamic magnification factor, Multiple tuned mass damper; Optimum parameters; Robustness; Vibration control

Received: 07 September 2018; Accepted: 02 October 2018

ISSN: 2630-5763 (online) C 2018 Golden Light Publishing® All rights reserved.

\section{Introduction}

The tuned mass damper (TMD) is composed of a mass, spring and viscous damper attached to a primary system in order to control resonant vibrations. Thus, the natural frequency of the absorber system is tuned to a frequency close to the natural frequency of the primary system. When the external excitation is resonant with the primary structure, TMD is a beneficial vibration control device that reduces the structural vibration.

The first study into the design of a tuned mass damper is conducted by Den Hartog [1], who derived a closed form solution for minimizing the dynamic response of a undamped primary system under harmonic excitation installed with a TMD. Since then, optimization of a TMD for various types of excitations have been extensively studied by many researchers [2-9]. A majority of those studies are concerned with improving the design procedure of optimum the TMD parameters.

Above-mentioned studies are concerned with the tuning of a single TMD to a specific vibration mode. Errors in estimation the structural frequency or manufacturing the TMD result in detuning of the 
TMD from the frequency of the primary system. To overcome the detuning problem, the use of multiple tuned mass dampers with differing dynamic characteristics is first proposed by $\mathrm{Xu}$ and Igusa [10]. Then, MTMD systems also studied and improved by many investigators [11-20]. These studies show that MTMD device have a wider frequency range than a TMD, thus it provides better effectiveness and higher robustness than a TMD in suppressing dynamic vibrations.

In spite of several studies on the optimization of the MTMD under harmonic excitation, the effects of manufacturing type on the optimum parameters, effectiveness and robustness of the MTMD system have not yet been studied. Thus, the five practical MTMD models are considered which consist of different combinations of the mass, damping, stiffness coefficient and damping ratio in the MTMD. The objective of this study is to present and discuss the performance of these five practical MTMD models under the same external harmonic excitation in order to give a reference to select the MTMD. Here, the optimum parameters of the MTMD system for damped primary system are presented. The primary system is represented by a single-degree-of-freedom system which corresponds to a particular vibration mode to be controlled in a real system. The criterion selected for optimality is minimization of the maximum value of displacement DMF of the primary system. The optimum parameters of the MTMD system are obtained for different mass ratios and number of TMD units of the MTMD using Sequential Quadratic Programming (SQP).

\section{Modeling of structure-MTMD system}

The system configuration consists of a primary system attached by $n$ tuned mass dampers with different dynamic characteristics as shown in Fig. 1. The primary system is characterized by the stiffness $k_{s}$, damping $c_{s}$, and mass $m_{s}$. The primary system and each TMD are modeled as SDOF system, thus, the total degrees of freedom of the structural system is $n+1$. The equations of motion for the primary system with MTMD subjected to harmonic excitation can be expressed as follows:

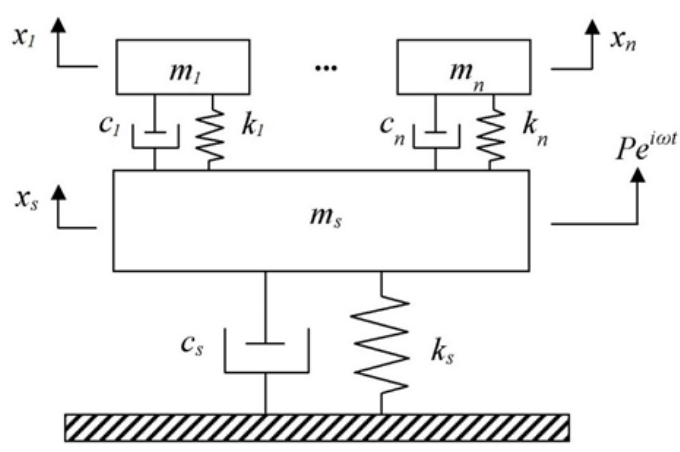

Fig. 1. Primary structure - MTMD coupled system $m_{s} \ddot{x}_{s}+c_{s} \dot{x}_{s}+k_{s} x_{s}+\sum_{j=1}^{n} c_{j}\left(\dot{x}_{s}-\dot{x}_{j}\right)+k_{j}\left(x_{s}-x_{j}\right)=P e^{i \omega t}$

$m_{j} \ddot{x}_{j}+c_{j}\left(\dot{x}_{j}-\dot{x}_{s}\right)+k_{j}\left(x_{j}-x_{s}\right)=0 \quad j=1,2, \cdots, n$

where over dot denotes differentiation with respect to time $t . m, c$ and $k$ are the mass, damping coefficient and stiffness, respectively. Subscripts $s$ and $j$ denote the primary structure and the $j$ th TMD, respectively. $x_{s}$ and $x_{j}$ indicate the vertical displacements.

Combining Eqs. (1) and (2), the equations of motion can be given in the following matrix form:

$$
\mathbf{M X}+\mathbf{C X}+\mathbf{K X}=\mathbf{F}
$$

where $\mathbf{M}, \mathbf{C}, \mathbf{K}$ are the mass, damping and stiffness matrices, $\ddot{\mathbf{X}}, \dot{\mathbf{X}}$ and $\mathbf{X}$ are the acceleration, velocity and displacement vectors, respectively, $\mathbf{F}$ is the external force vector, that can be defined as:

$$
\begin{aligned}
& \mathbf{M}=\operatorname{diag}\left(m_{s} \quad m_{1} \quad m_{2} \quad \cdots \quad m_{n}\right] \\
& \mathbf{C}=\left[\begin{array}{ccccc}
c_{s}+\sum_{j=1}^{n} c_{j} & -c_{1} & -c_{2} & \cdots & -c_{n} \\
-c_{1} & c_{1} & 0 & \cdots & 0 \\
-c_{2} & 0 & c_{2} & \cdots & 0 \\
\vdots & \vdots & \vdots & \ddots & \vdots \\
-c_{n} & 0 & 0 & \cdots & c_{n}
\end{array}\right]
\end{aligned}
$$


$\mathbf{K}=\left[\begin{array}{ccccc}k_{s}+\sum_{j=1}^{n} k_{j} & -k_{1} & -k_{2} & \cdots & -k_{n} \\ -k_{1} & k_{1} & 0 & \cdots & 0 \\ -k_{2} & 0 & k_{2} & \cdots & 0 \\ \vdots & \vdots & \vdots & \ddots & \vdots \\ -k_{n} & 0 & 0 & \cdots & k_{n}\end{array}\right]$

$\mathbf{X}=\left\{\begin{array}{lllll}x_{s} & x_{1} & x_{2} & \cdots & x_{n}\end{array}\right\}^{\mathrm{T}}$,

$\mathbf{F}=e^{i \omega t}\left\{\begin{array}{lllll}P & 0 & 0 & \cdots & 0\end{array}\right\}^{\mathrm{T}}$

The amplitude of the displacement of the primary system is expressed in the normalized form as

$$
R=\sqrt{\frac{1}{\operatorname{Re}\left(z_{1}\right)^{2}+\operatorname{Im}\left(z_{1}\right)^{2}}}
$$

and

$$
\begin{aligned}
& \operatorname{Re}\left(z_{1}\right)=1-\beta^{2}-\beta^{2} \sum_{j=1}^{n} \mu_{j} \frac{\frac{\beta_{j}^{2}}{\beta^{2}}-1+4 \xi_{j}^{2}}{\left(\frac{\beta_{j}}{\beta}-\frac{\beta}{\beta_{j}}\right)^{2}+4 \xi_{j}^{2}}, \\
& \operatorname{Im}\left(z_{1}\right)=2 \beta \xi_{s}+\beta^{2} \sum_{j=1}^{n} \mu_{j} \frac{\frac{2 \beta \xi_{j}}{\beta_{j}}}{\left(\frac{\beta_{j}}{\beta}-\frac{\beta}{\beta_{j}}\right)^{2}+4 \xi_{j}^{2}}
\end{aligned}
$$

where $x_{s t}=P / k_{s}$ is the static displacement of the structure, $\beta=\omega / \omega_{s}$ is the frequency ratio between the external force and the structure, $\xi_{s}$ is the damping ratio of the structure, $\mu_{j}=m_{j} / m_{s}$ is the mass ratio, $\beta_{j}=\omega_{j} / \omega_{s}$ is the frequency ratio, and $\xi_{j}=c_{j} / 2 m_{j} \omega_{j}$ is the damping ratio of $j$ th TMD. Details can be found in $\mathrm{Li}$ and $\mathrm{Ni}$ [15].

The natural frequency $\omega_{j}$ (i.e., $\omega_{j}=\sqrt{k_{j} / m_{j}}$ ) $j$ th TMD is presented by

$$
\omega_{j}=\omega_{T}\left[1+\left(j-\frac{n+1}{2}\right) \frac{\psi}{n-1}\right]
$$

and
$\omega_{T}=\sum_{j=1}^{n} \omega_{j} / n$

$\psi=\frac{\omega_{n}-\omega_{1}}{\omega_{T}}$

where $\omega_{T}$ is the average frequency of all MTMD and $\psi$ is the non-dimensional frequency spacing of the MTMD.

Total mass of the MTMD system is expressed by the mass ratio defined as

$\mu=\frac{\sum_{j=1}^{n} m_{j}}{m_{s}}$

where $\mu$ is the mass ratio of the MTMD system.

Tuning frequency ratio of the MTMD system is expressed by

$f=\frac{\omega_{T}}{\omega_{s}}$

where $\omega_{s}$ (i.e., $\omega_{s}=\sqrt{k_{s} / m_{s}}$ ) is the natural frequency of the primary system.

The average damping ratio of the MTMD is expressed as

$\xi_{T}=\sum_{j=1}^{n} \xi_{j} / n$

and

$\xi_{j}=\frac{c_{j}}{2 m_{j} \omega_{j}}$

where $\xi_{j}, c_{j}, m_{j}$ and $\omega_{j}$ are the damping ratio, damping coefficient, mass and natural frequency of $j$ th TMD unit, respectively.

To illustrate the effects of manufacturing type on control performance of multiple tuned mass dampers for reducing undesirable vibration under the harmonic excitation, five different MTMD models are used in the following sections. Details in the considered MTMD models can be found in Li 2002 [21]. For different MTMDs, the 
expressions for the quantities $\mu_{j}$ and $\xi_{j}$ are given in Table 1.

\section{Numerical application}

In this section, an optimization problem optimization is defined as minimization of Eq. (8) as follows:

$\min J(q)$ with $\quad l_{b} \leq q \leq u_{b}$

where $q, J(q), l_{b}$ and $u_{b}$ represent the optimization variables, the objective function, the lower and upper bounds of the optimization variables, respectively. The optimization variables are as follows:

$$
q=\left[\xi_{T}, f, \psi\right]
$$

The ranges are selected for the design parameters: $0<\xi_{T}<0.5,0.5<f<1.5,0<\psi<0.5$, with the search increment of each parameter set to be 0.001 . The range of excitation frequency ratio is also assumed to be $0.5<\beta<1.5$ Unfortunately, it is not easy to obtain an explicit solution for the optimal parameters analytically from Eq. (8). Thus, a numerical optimization is performed using the MATLAB fmincon function. SQP is a powerful nonlinear optimization algorithm in order to solve the above mentioned design problem. SQP is inbuilt fmincon function in Matlab. Firstly, the system parameter is defined, and initial values and boundaries for tuning parameters are set.

Then, Eq. (8) is calculated numerically. The results are used to find the objective function. After, the fmincon command is applied to update all parameters. The final step is to check whether the objective function is minimum or not. If the objective function is minimized, then the optimized set of parameters is obtained and the optimization problem has been successfully solved. Otherwise, the updated set of parameters should be used to solve Eq. (8) again and calculate the objective function. This process should be repeated until the optimized set of parameters is achieved.

To verify the above optimization technique, firstly the obtained optimum parameters and response amplitudes are compared with the literature results for undamped primary system. It can be observed from Table 2 that the present results are in good agreement with the literature results for optimum values. The maximum difference between the results is also found to be less than $2.5 \%$.

Table 1. Expressions for the quantities $\mu_{j}$ and $\xi_{j}$ for different MTMD models

\begin{tabular}{|c|c|c|c|}
\hline MTMD type & System parameters & $\mu_{j}$ & $\xi_{j}$ \\
\hline MTMD-1 & $\begin{array}{l}m_{1} \neq m_{2} \neq \ldots \neq m_{n}, c_{1}=c_{2}=\ldots=c_{n} \\
k_{1}=k_{2}=\ldots=k_{n}, \xi_{1} \neq \xi_{2} \neq \ldots \neq \xi_{n}\end{array}$ & $\mu / \beta_{j}^{2} \sum_{j=1}^{n}\left(1 / \beta_{j}^{2}\right)$ & $\beta_{j} \xi_{T}$ \\
\hline MTMD-2 & $\begin{array}{l}m_{1}=m_{2}=\ldots=m_{n}, c_{1} \neq c_{2} \neq \ldots \neq c_{n} \\
k_{1} \neq k_{2} \neq \ldots \neq k_{n}, \xi_{1}=\xi_{2}=\ldots=\xi_{n}\end{array}$ & $\mu / n$ & $\xi_{T}$ \\
\hline MTMD-3 & $\begin{array}{l}m_{1}=m_{2}=\ldots=m_{n}, c_{1}=c_{2}=\ldots=c_{n} \\
k_{1} \neq k_{2} \neq \ldots \neq k_{n}, \xi_{1} \neq \xi_{2} \neq \ldots \neq \xi_{n}\end{array}$ & $\mu / n$ & $n \xi_{T} / \beta_{j} \sum_{j=1}^{n}\left(1 / \beta_{j}\right)$ \\
\hline MTMD-4 & $\begin{array}{l}m_{1} \neq m_{2} \neq \ldots \neq m_{n}, c_{1} \neq c_{2} \neq \ldots \neq c_{n} \\
k_{1}=k_{2}=\ldots=k_{n}, \xi_{1}=\xi_{2}=\ldots=\xi_{n}\end{array}$ & $\mu / \beta_{j}^{2} \sum_{j=1}^{n}\left(1 / \beta_{j}^{2}\right)$ & $\xi_{T}$ \\
\hline MTMD-5 & $\begin{array}{l}m_{1} \neq m_{2} \neq \ldots \neq m_{n}, c_{1}=c_{2}=\ldots=c_{n} \\
k_{1} \neq k_{2} \neq \ldots \neq k_{n}, \xi_{1}=\xi_{2}=\ldots=\xi_{n}\end{array}$ & $\mu / \beta_{j} \sum_{j=1}^{n}\left(1 / \beta_{j}\right)$ & $\xi_{T}$ \\
\hline
\end{tabular}


Table 2. Optimal MTMD parameters for various numbers of TMD units ( $\mu=0.01)$

\begin{tabular}{clllll}
\hline \multirow{2}{*}{ Optimum values } & $n=5$ & & $n=15$ & \\
\cline { 2 - 3 } \cline { 5 - 6 } & Present study & Ref. [17] & & Present study & Ref. [17] \\
\hline$R$ & 11.445 & 11.446 & 11.158 & 11.158 \\
$f$ & 0.9957 & 0.9957 & & 0.9970 & 0.9970 \\
$\psi$ & 0.1118 & 0.1113 & & 0.1397 & 0.1399 \\
$\xi_{T}$ & 0.0239 & 0.0239 & & 0.0166 & 0.0162 \\
\hline
\end{tabular}

\section{Numerical results}

To investigate the difference between the optimum parameters and control performance of MTMD models, several numerical analyses are performed and discussed in following sections. The damping ratio of primary structure is assumed to be 0.02 . Firstly, the variations in the optimum parameters and optimum $R$ against the mass ratio are presented considering the different values of the total number in Figs. 2-5. Then, the variations in the optimum parameters and optimum $R$ against the number of TMD units are presented considering the different values of the mass ratio in Figs. 6-9.

The change of the optimum frequency spacing with respect to the total number for the five MTMD models in shown in Fig. 2, for the two cases: (a) $\mu=0.01$ and (b) $\mu=0.05$. From Fig. 2 , it is observed that the optimum values of the frequency spacing increase with the increase of both the mass ratio and total number for five MTMD models. It is seen also from these figures that with the increase of number of MTMD the frequency spacing increases but when the total number is beyond a certain value (in this case $n=12$ ) the increase in the optimum frequency spacing stays almost stationary. Compared with the other curves of the optimum frequency spacing curve in these figures, as can also be seen, the optimum values of the frequency spacing for the optimum MTMD-1 and MTMD-5 are very similar. In Fig. 2(a), it also observed that the difference in the optimum values of the frequency spacing for five optimum MTMDs is almost undetectable when the total number is less than 8 .

The change of the optimum tuning frequency ratio with respect to the total number is illustrated in Fig. 3 for the five MTMD models, for the two cases: (a) $\mu=0.01$ and (b) $\mu=0.05$. It can be shown that, for the optimum MTMD-1, MTMD-4 and MTMD-5, the optimum tuning frequency ratio generally increases with an increase in the total number and decreases with the increasing of the mass ratio. However, the optimum tuning frequency ratio reveals the irregular variation type for the optimum MTMD-2 and MTMD-3.

The relationship of the optimum average damping ratio with respect to the total number for the five MTMD models for the two cases: (a) $\mu=$ 0.01 and (b) $\mu=0.05$, is shown in Fig.4. It is shown in Fig. 4 that the optimum average damping ratio increases with the increasing of the mass ratio and decreases with an increase in the total number. However, the MTMD-2 and MTMD-3 provide the higher values of the optimum damping ratio for larger mass ratio when the total number is larger than or equal to 8 .

The optimum value of $R$ is plotted against the total number for the five MTMD models for the two cases: (a) $\mu=0.01$ and (b) $\mu=0.05$, as shown in Fig. 5. It can be seen that the total number of MTMDs is most important factor on the effectiveness of the MTMD. However, when the total number of MTMDs is increased beyond a certain number (in this case $n=12$ ) for both mass ratios, little further effect on structure response is shown. Furthermore, from these figures, the optimum MTMD-1 and MTMD-4 provides better effectiveness than all the other types of MTMD of the same mass ratio in suppressing structural vibrations. 


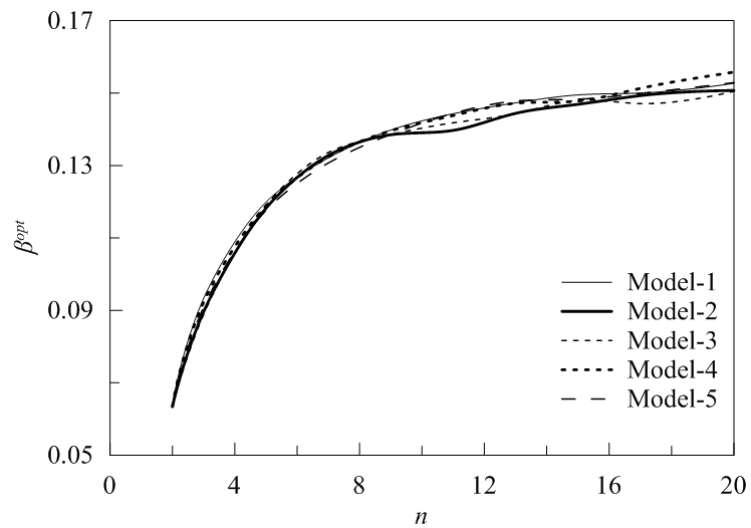

a) $\mu=0.01$

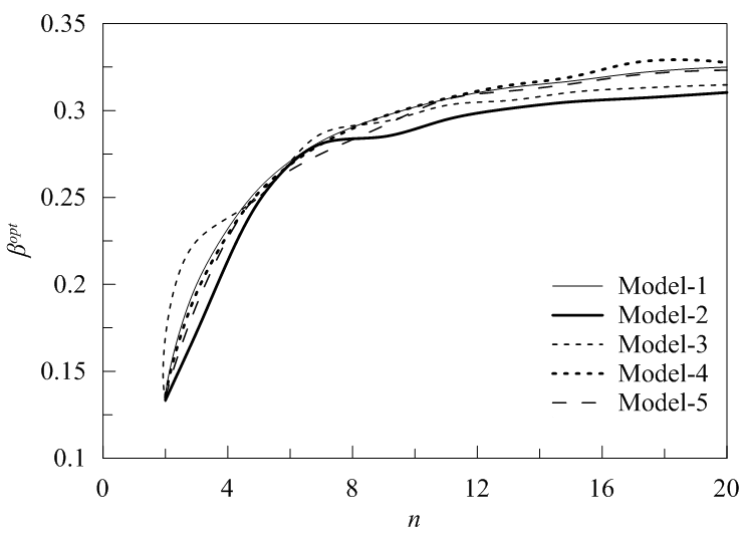

b) $\mu=0.05$

Fig. 2. $\beta^{\text {opt }}-n$ relationship of five MTMD models

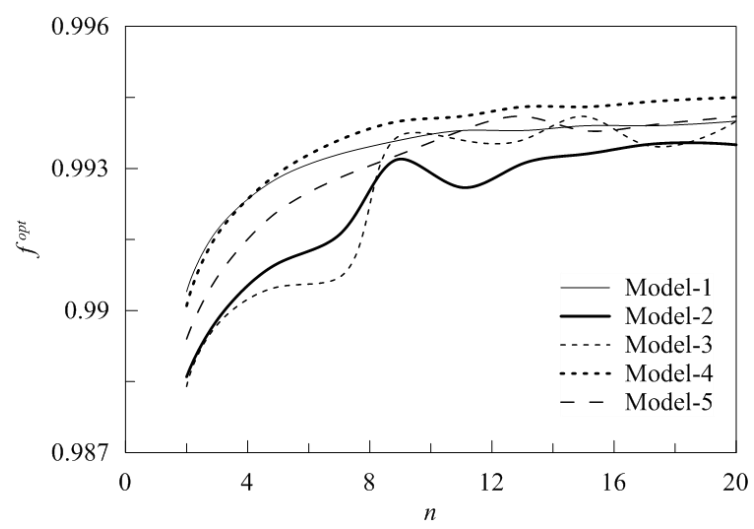

a) $\mu=0.01$

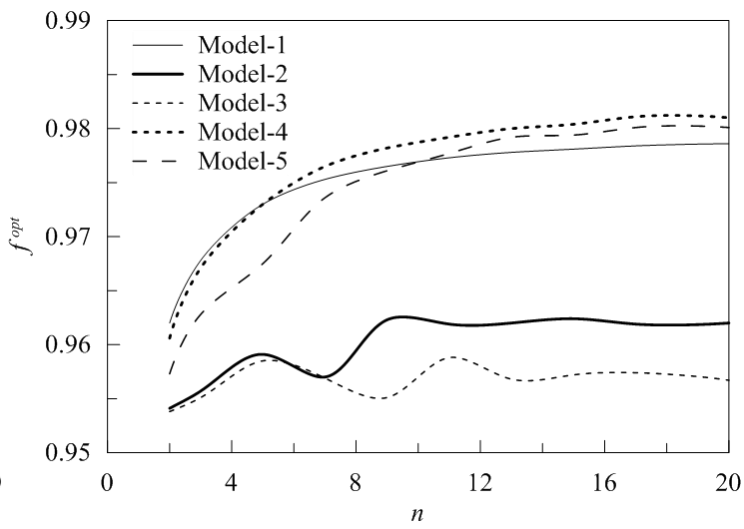

b) $\mu=0.05$

Fig. 3. $f^{o p t}-n$ relationship of five MTMD models

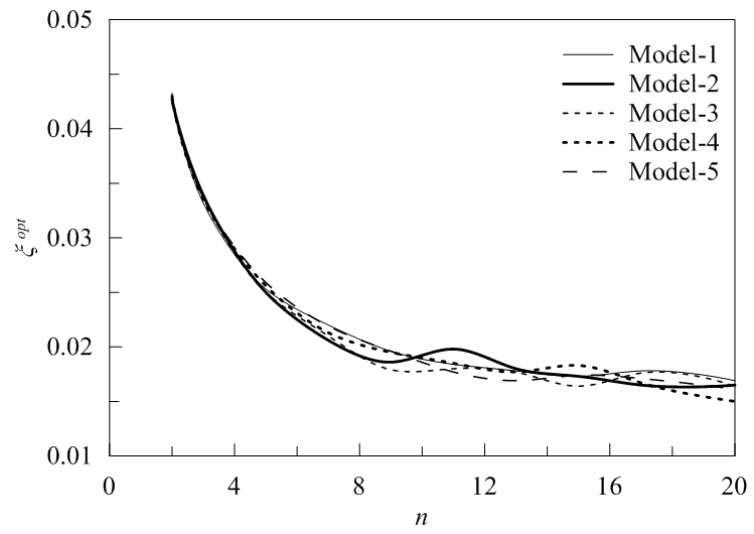

a) $\mu=0.01$

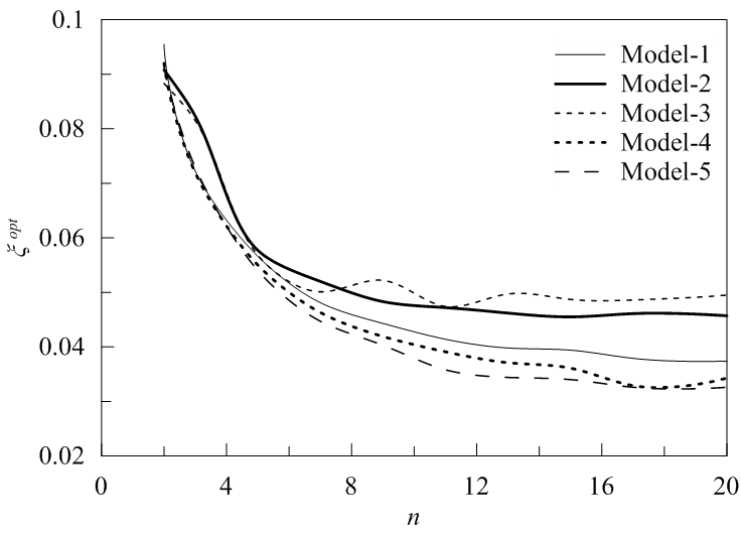

b) $\mu=0.05$

Fig. 4. $\xi^{\text {opt }}$ - $n$ relationship of five MTMD models 


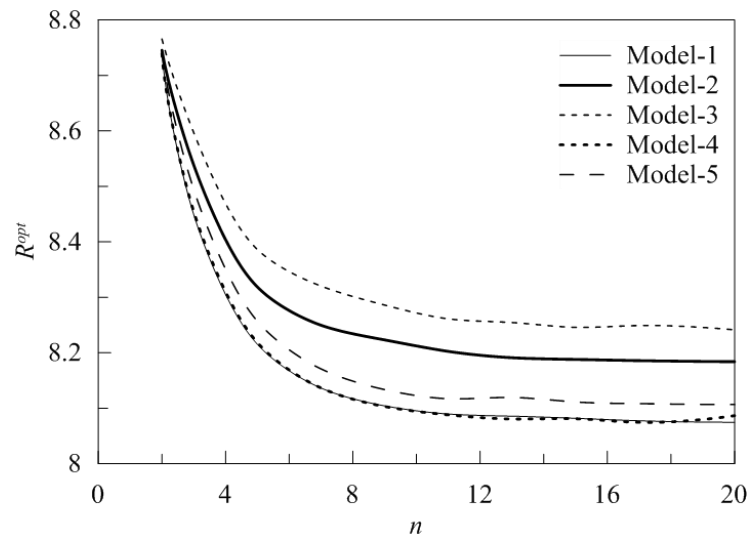

a) $\mu=0.01$

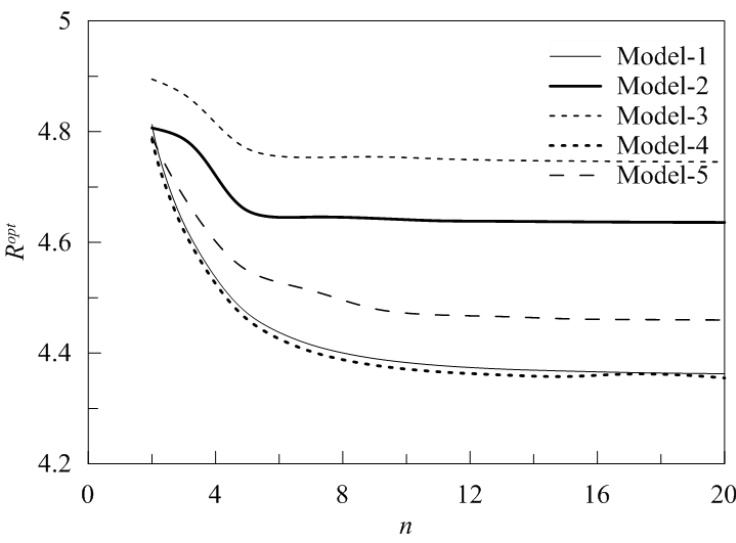

b) $\mu=0.05$

Fig. 5. $R^{\text {opt }}-n$ relationship of five MTMD models

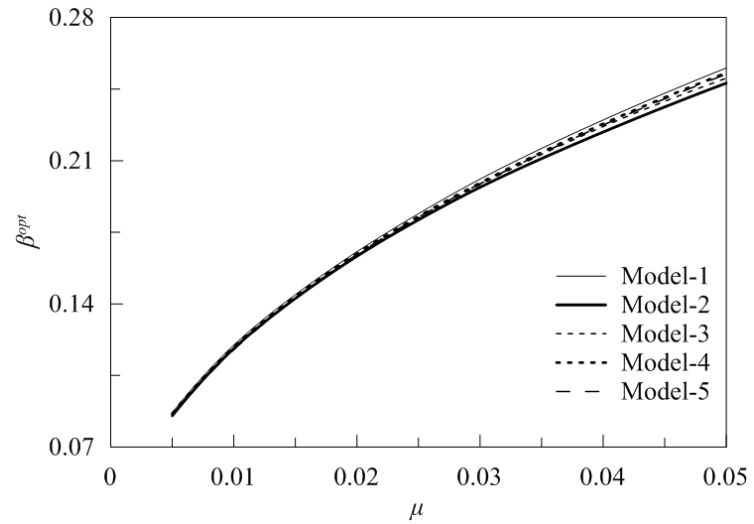

a) $n=5$

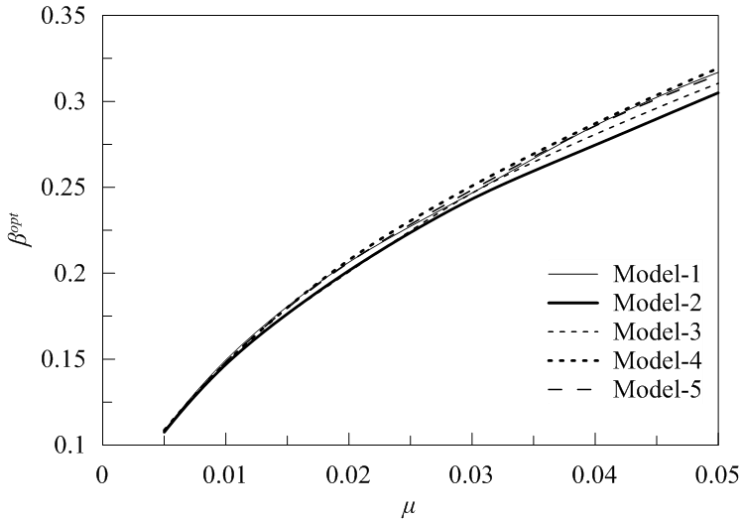

b) $n=15$

Fig. 6. $\beta^{\text {opt }}-\mu$ relationship of five MTMD models

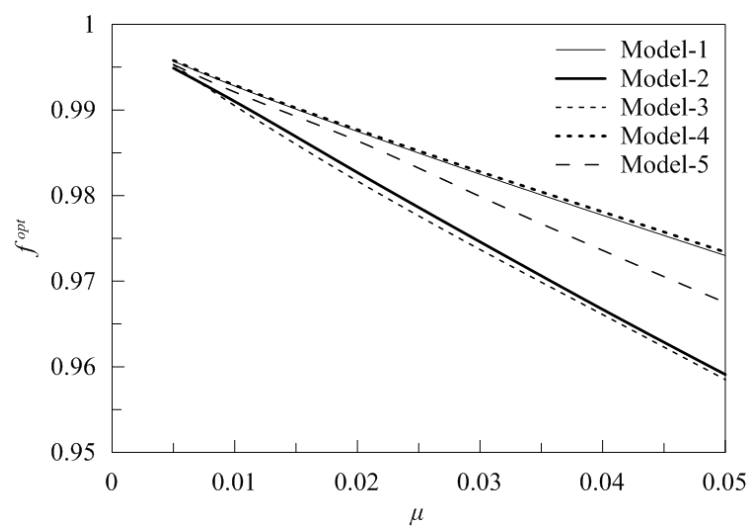

a) $n=5$

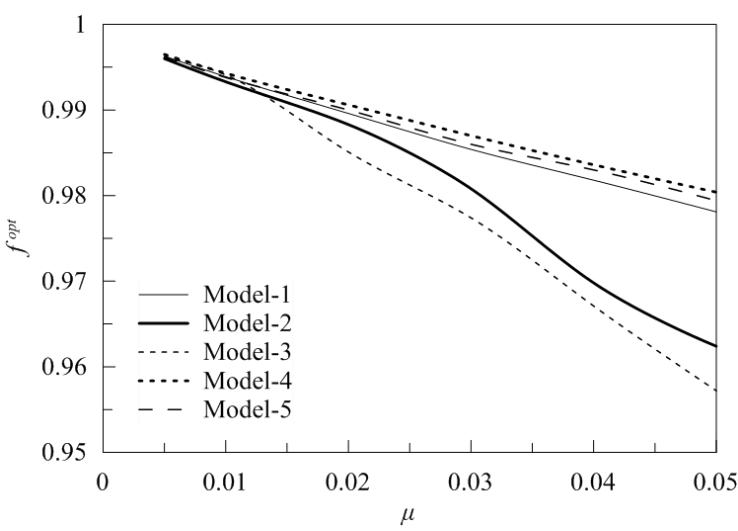

b) $n=15$

Fig. 7. $f^{o p t}-\mu$ relationship of five MTMD models 


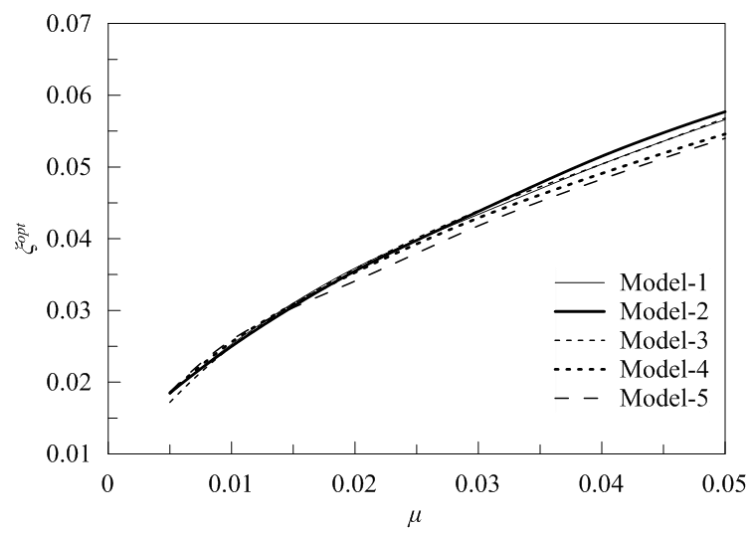

a) $n=5$

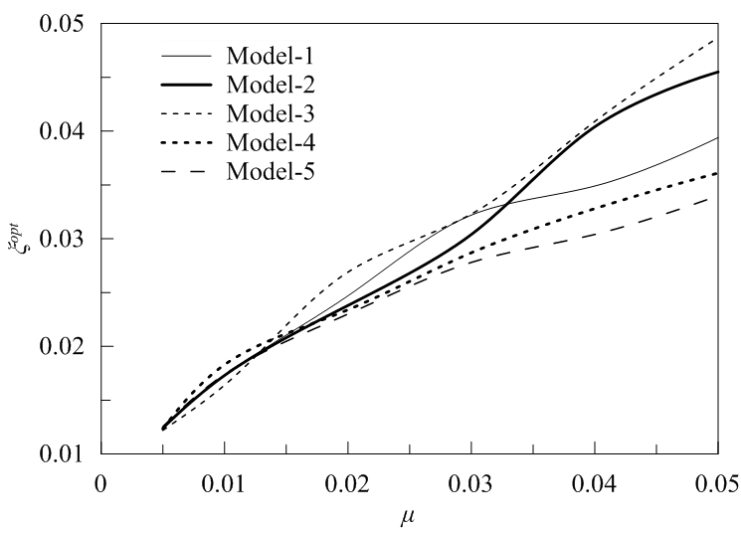

b) $n=15$

Fig. 8. $\xi^{o p t}-\mu$ relationship of five MTMD models

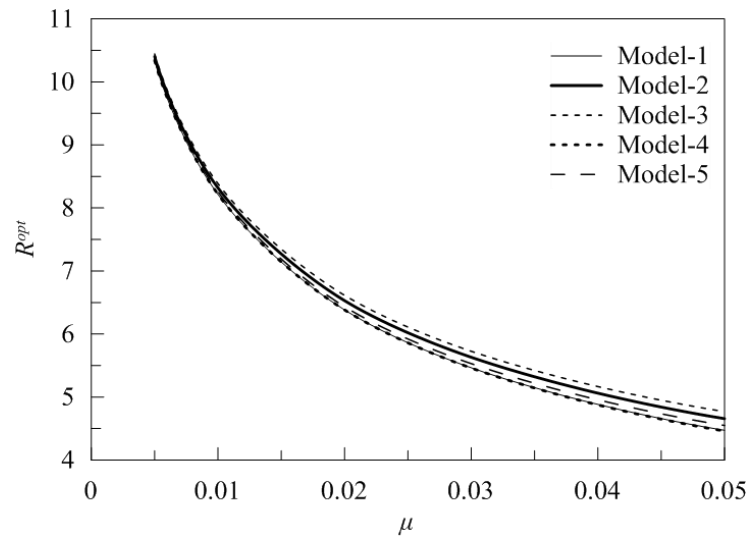

a) $n=5$

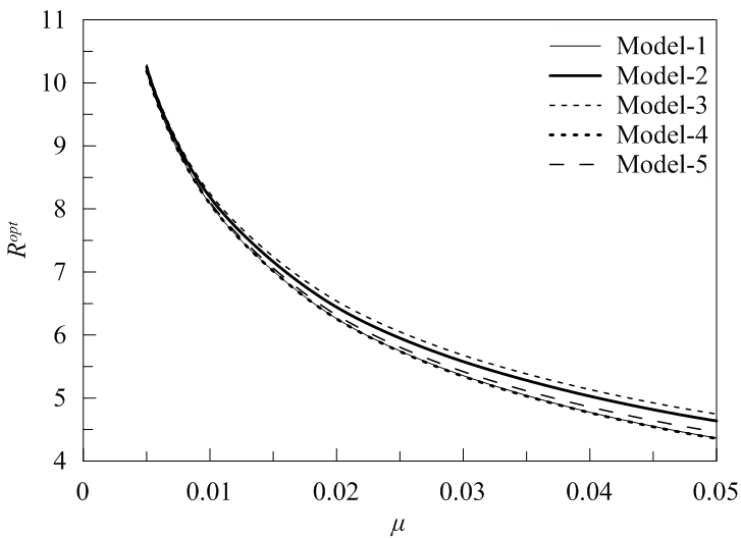

b) $n=15$

Fig. 9. $R^{\text {opt }}-\mu$ relationship of five MTMD models

The variation of the optimum frequency spacing of the MTMD system versus the mass ratio is illustrated in Fig. 6 for the two cases: (a) $n=5$ and (b) $n=15$. As the mass ratio increases, the optimum frequency spacing increases. The difference in the optimum frequency spacing between five MTMD models increases slightly with the increase of mass ratio. It is also observed that the optimum frequency spacing of the MTMD system increases with an increase in both the mass ratio and the total number of MTMDs.

The variation of the optimum tuning frequency ratio of the MTMD system versus the mass ratio is illustrated in Fig. 7 for the two cases: (a) $n=5$ and (b) $n=15$. From these figures, it observed that with the increase of mass ratio of MTMD the optimum tuning frequency ratio decreases whereas with the increase of total number of MTMD the optimum tuning frequency ratio increases. The difference in the optimum tuning frequency ratio between five MTMD models increases apparently with the increase of mass ratio. In addition, the optimum tuning frequency ratio for MTMD-2 and MTMD-3 is smaller than the other MTMD systems.

In Fig. 8 is shown the optimum average damping ratio of the MTMD system versus the mass ratio for the two cases: (a) $n=5$ and (b) $n=$ 15. The optimum average damping ratio increases with an increase in the mass ratio and decreases with an increase in the total number of MTMD. Furthermore, for high values of the mass ratio, the optimum average damping ratio of the MTMD-2 
and MTMD-3 systems is also greater compared to that of the optimum other MTMD systems.

In Fig. 9 is shown the optimum value of $R$ versus the mass ratio for the two cases: (a) $n=5$ and (b) $n=15$. As can be seen from these figures, the displacement of the primary system decreases with the increase of the mass ratio of the MTMD system. It is also observed that the optimum MTMD-1 and MTMD-4 practically provides the same effectiveness. Another observation from Fig. 9 is that the optimum MTMD-5 attains nearly the same effectiveness as the optimum MTMD-1 and MTMD-4. Thus, the optimum MTMD-1, MTMD4, and MTMD-5 provides better effectiveness.

$R$ curves for all cases are plotted against the excitation frequency ratio normalized by the structural natural frequency as shown in Fig. 10. It is observed that MTMD-1 and MTMD-4 provide superior vibration suppression performance comparing to that of the other cases. However, compared with the other control models, MTMD-1 and MTMD-4 also provides smooth performance in a narrow frequency range centered at the tuned frequency.

In real structures, the control performance of MTMDs can be significantly detuned when there are some errors in the identifying the structural frequency or manufacturing the MTMD. Thus, the robustness of MTMD against error in the tuning frequency ratio is also investigated and the results are shown in Fig. 11. The abscissa is the error in the optimal tuning frequency ratio while the ordinate is the maximum $R$. As can be seen that the effectiveness of MTMD- 1 and MTMD- 4 are better than that of the other cases when the MTMD system is optimal. However, it is also observed that MTMD-1 and MTMD-4 provides better effectiveness than the other cases at the range of the errors from $-5 \%$ to $+2 \%$. But MTMD-1 and MTMD-4 provides worse effectiveness with respect to the rest of the MTMD models when the error is beyond $+2 \%$.

\section{Conclusions}

In this paper, the optimum parameters of five MTMD models with the damped primary structure under external harmonic excitation are presented in detail. The optimum parameters of the MTMD model (i.e., the damping ratio, tuning frequency ratio and frequency spacing) are found using SQP method based on mass ratio and number of the MTMD. The performances of these five MTMD models have been also investigated through comprehensive numerical analyses based on detuning resulting from a frequency deviation in MTMD device. The following conclusions can be drawn from the present study:

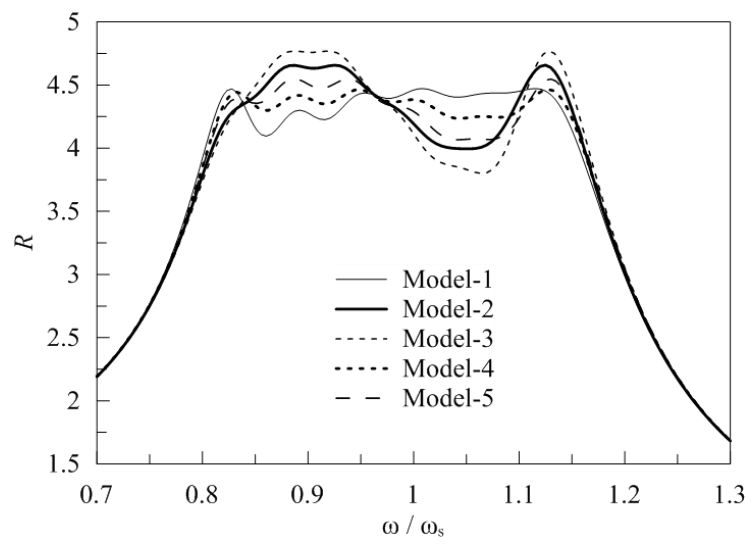

a) $n=5, \mu=0.05$

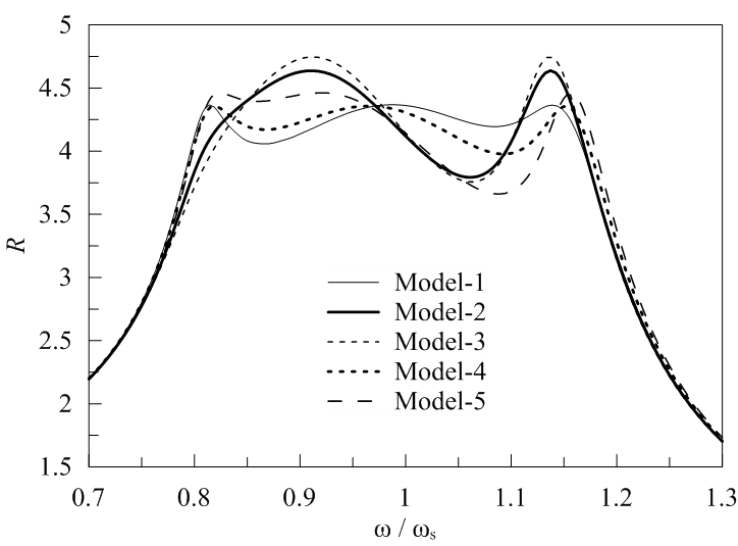

b) $n=15, \mu=0.05$

Fig. 10. Frequency response curves of the primary system with five MTMD models 


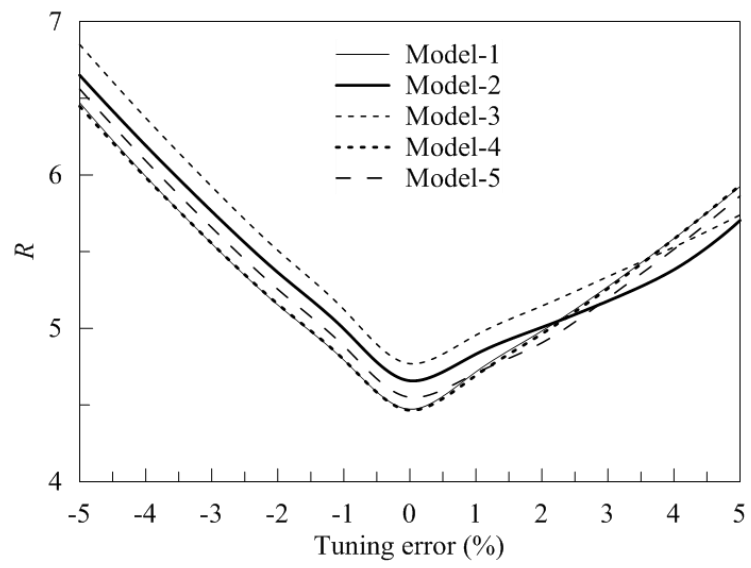

a) $n=5$

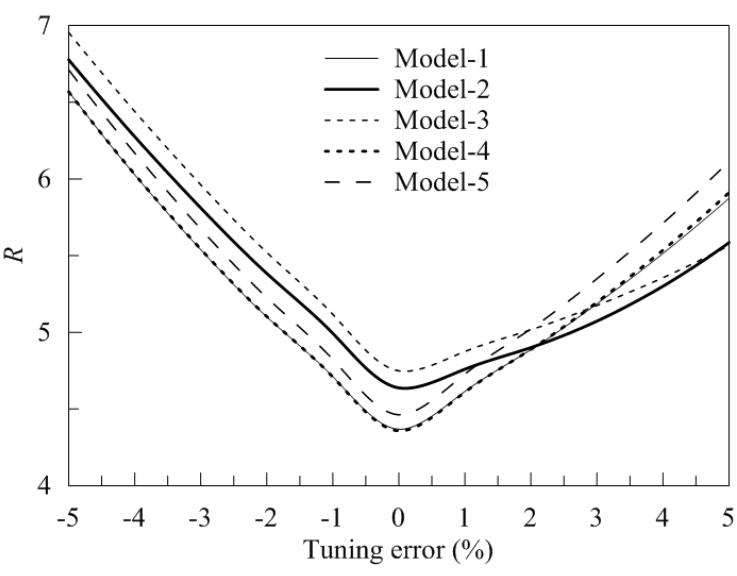

b) $n=15$

Fig. 11. Robustness of MTMD systems to variations in the tuning frequency

1. The optimum damping ratio and response of the primary system decreases with the increase of number of MTMD while the optimum frequency spacing and tuning frequency ratio increases.

2. The tuning frequency ratio and response of the primary system decreases with the increase of mass ratio of MTMD while the optimum frequency spacing and damping ratio increases.

3. The optimum MTMD-1 and MTMD-4 gain nearly the same control performance, and provide the better effectiveness than the optimum MTMD-2, MTMD-3 and MTMD-5.

4. The robustness of MTMD-1 and MTMD-4 are generally better than the other cases when the change of the frequency tuning ratio is small.

5. The optimum MTMD-1 can be preferred in the other control devices when the manufacturability, effectiveness and robustness are considered.

6. To overcome the problems of the detuning, the errors of estimate of the parameters of the structure or MTMD system are should be considered in the design process of MTDM devices.

\section{References}

[1] Den Hartog JP. Mechanical vibrations, McGrawHill, New York, 1956.
[2] Tsai HC, Lin GC (1993) Optimum tuned-mass dampers for minimizing steady-state response of support-excited and damped systems, Earthquake Engineering \& Structural Dynamics 22(11): 957-973.

[3] Wu JJ (2006) Study on the inertia effect of helical spring of the absorber on suppressing the dynamic responses of a beam subjected to a moving load. Journal of Sound and Vibration. 297(3-5): 981-999.

[4] Bakre SV, Jangid RS (2007) Optimum parameters of tuned mass damper for damped main system. Structural Control and Health Monitoring. 14(3): 448-470.

[5] Leung AYT, Zhang H (2009) Particle swarm optimization of tuned mass dampers. Engineering Structures 31(3): 715-728.

[6] Bekdaş G, Nigdeli SM (2013) Mass ratio factor for optimum tuned mass damper strategies. International Journal of Mechanical Sciences 71: 68-84.

[7] Özsarıyıldız ŞS, Bozer A (2015) Finding optimal parameters of tuned mass dampers. The Structural Design of Tall and Special Buildings. 24(6): 461-475.

[8] Nigdeli SM, Bekdaş G (2017) Optimum tuned mass damper design in frequency domain for structures, KSCE Journal of Civil Engineering 21(3): 912-922.

[9] Dell'Elce L, Gourc E, Kerschen G (2018) A robust equal-peak method for uncertain mechanical systems. Journal of Sound and Vibration 414: 97-109. 
[10] Xu K, Igusa T (1992) Dynamic characteristics of multiple substructures with closely spaced frequencies. Earthquake Engineering \& Structural Dynamics 21(12): 1059-1070.

[11] Joshi AS, Jangid RS (1997) Optimum parameters of multiple tuned mass dampers for base-excited damped systems. Journal of Sound and Vibration 202(5): 657-667.

[12] Jangid RS (1999) Optimum multiple tuned mass dampers for base-excited undamped system. Earthquake Engineering \& Structural Dynamics 28(9): 1041-1049.

[13] Park J, Reed D (2001) Analysis of uniformly and linearly distributed mass dampers under harmonic and earthquake excitation. Engineering Structures 23(7): 802-814.

[14] Poovarodom N, Kanchanosot S, Warnitchai P (2003) Application of non-linear multiple tuned mass dampers to suppress man-induced vibrations of a pedestrian bridge. Earthquake Engineering \& Structural Dynamics 32(7): 11171131.

[15] Li HN, Ni XL (2007) Optimization of nonuniformly distributed multiple tuned mass damper. Journal of Sound and Vibration 308: 8097.

[16] Lin CC, Wang JF, Lien CH, Chiang HW, Lin CS (2010) Optimum design and experimental study of multiple tuned mass dampers with limited stroke. Earthquake Engineering \& Structural Dynamics 39(14): 1631-1651.
[17] Bandivadekar TP, Jangid RS (2013) Optimization of multiple tuned mass dampers for vibration control of system under external excitation. Journal of Vibration and Control: SAGE 19(12): 1854-1871.

[18] Yang F, Sedaghati R, Esmailzadeh E (2015) Optimal design of distributed tuned mass dampers for passive vibration control of structures. Structural Control and Health Monitoring 22(2): 221-236.

[19] Yazdi HA, Saberi H, Saberi H, Hatami F (2016) Designing optimal tuned mass dampers using improved harmony search algorithm. Advances in Structural Engineering 19(10): 1620-1636.

[20] Bozer A, Özsarıyıldız ŞS (2018) Free parameter search of multiple tuned mass dampers by using artificial bee colony algorithm. Structural Control and Health Monitoring 25(2) https://doi.org/10.1002/stc.2066.

[21] Li C (2002) Optimum multiple tuned mass dampers for structures under the ground acceleration based on DDMF and ADMF. Earthquake Engineering \& Structural Dynamics 31: 897-919. 